\title{
Dispersion of Transverse Waves in Single-Wall Carbon Nanotubes
}

\author{
M.M. SELIM ${ }^{a, b, *}$ \\ ${ }^{a}$ Department of Mathematics, College of Science and Humanities in Al-Aflaj, \\ Prince Sattam bin Abdulaziz University, Al-Aflaj 710-11912, Saudi Arabia \\ ${ }^{b}$ Department of Mathematics, Suez Faculty of Science, Suez University, Egypt
}

(Received October 3, 2019; in final form October 10, 2019)

\begin{abstract}
In this work we investigate the influence of initial stress on the transverse waves propagation in single-wall carbon nanotubes with small scale effects under ultrahigh frequency (above $k=1 \mathrm{~nm}^{-1}$ ), using the nonlocal continuum theory. The phase and group velocities are derived in a simple way. The effects of wave numbers, scale coefficients and initial stress parameter on phase and group velocity are analyzed. The results show that the dispersion properties of the transverse wave propagation in a single-wall carbon nanotube are induced by small scale effects, which cannot appear in classical models. Also, it is clear that the increase of the scale coefficient and wave number could be strengthened by the dispersion degree of the phase and group velocity of transverse waves. In particular, the analysis shows that the compression initial stress in a single-wall carbon nanotube does not only affect the number of transverse wave speeds and the magnitude of transverse wave speeds, but also the critical value of the wave number at the wave speed changes. The effects of the initial stress and small scale on the transverse wave propagation in single-wall carbon nanotubes may result in some complex dynamic phenomena of terahertz sound waves propagation in the carbon nanotubes, which can be used as a useful reference for the designs of nanodrive devices, nanooscillators and nanosensors.
\end{abstract}

DOI: 10.12693/APhysPolA.136.1018

PACS/topics: single-wall carbon nanotubes, transverse waves, Erigena's nonlocal elasticity theory, initial stresses, small scale effect

\section{Introduction}

The carbon nanotubes possess remarkable mechanical and physical properties [1-4] leading to many potential applications such as fluid transport, fluid storage at nanoscale, and nanodevices for drug delivery $[5,6]$. In addition to experimental endeavors, CNT modeling is classified into two main categories. The first is atomic modeling, which includes such techniques as classical molecular dynamics (MD) and tight-binding MD and the density functional model [7-11]. Li and Chou [12] reported an atomistic simulation of single-walled carbon nanotube (SWCNT) subjected to harmonic waves. Atomic modeling is limited to systems with a small number of molecules and atoms and is therefore confined to smallscale modeling. The second category is continuum modeling [13-16], which includes classical (or local) beam and shell theories that are practical for analyzing CNTs for large-scale systems. Successful work has been conducted with continuum modeling, such as buckling analysis, dynamics studies, and mechanical property investigations of CNTs [17-21].

Solid mechanics with elastic continuum model have been regarded as an effective method and widely used for studying the mechanical and physical properties of CNTs [22, 23]. The nanostructures length scales are often

*e-mail: m.selim@psau.edu.sa sufficiently small, and hence for the applicability of classical continuum models, we need to consider the small length scales such as lattice spacing between individual atoms, grain size, etc. The conventional continuum models cannot handle scale effects. Hence, the best alternative is to use those methods which provides the simplicity of continuum models and at the same time incorporate the effects of scale in such chosen continuum models. The Erigena nonlocal elasticity theory [24, 25] is a useful tool in treating phenomena whose origins lie in the regimes smaller than the classical continuum models. In this theory, the internal size or scale could be represented in the constitutive equations simply as material parameters. Such a nonlocal continuum mechanics has been widely accepted and has been applied to many problems including wave propagation, dislocation, crack problems, etc. [26]. In recent years, the application of nonlocal continuum mechanics in nanostructures such as carbon nanotubes (CNTs), graphene sheets and nanoplates has received a great interest [27]. On the other hand, the carbon nanotubes often suffer from initial stresses due to residual stress, thermal effect, surface effect, mismatch between the material properties of the nanotubes and a surrounding medium, initial external loads, and may be due to any other physical cause. In this field, the effects of initial stress on the noncoaxial resonance of multi-walled nanotubes (MWNTs) have been investigated by the theories of the EulerBernoulli and Timoshenko beams, respectively, in Wang and Cai [28] and Cai and Wang [29]. Zhang et al. [30] studied the transverse vibrations of DWNTs under 
compressive axial load using the Euler-Bernoulli beam theory. Lu et al. [31] adopted a nonlocal Euler-Bernoulli beam model to analyze wave and vibration characteristics of one-dimensional nanostructures with axially initial stress. Furthermore, Wang et al. [32] used a nonlocal Timoshenko beam model to deal with free vibration of micro- and nanobeams with initial stress. The vibrations of single- and multi-wall carbon nanotubes have been studied by various authors [33-37].

In this paper, we extend our study of waves propagating in carbon nanotubes. A model of transverse waves propagation in pre-stressed single-wall carbon nanotubes with small scale effects will be investigated in a simple way. The governing equations for transverse waves have been derived using Erigena's nonlocal elasticity theory. The numerical results are shown graphically.

\section{Mathematical formulation}

The local elasticity method is limited for practical calculation of nanotubes. However, in the nonlocal continuum theory presented by Eringen [24, 25], the stress at a reference point is considered as a function of the strain at every point in the body. Then, the long-range force of microstructures can be considered and are applied widely in fracture mechanics [38], contact mechanics [39] and dislocation theory [40]. The most general form of the constitutive equation for nonlocal elasticity involves an integral over the entire region of interest. This integral contains a kernel function that describes the relative influences of strains at various locations on the stress at a given location. The constitutive equations of linear, homogeneous, isotropic, nonlocal elastic solid with zero body forces can be written as follows [25]:

$$
\begin{aligned}
& \sigma_{i j, j}=0, \\
& \sigma_{i j}=\int \alpha\left(\left|x-x^{\prime}\right|, \tau\right) C_{i j k l} \varepsilon_{k l}\left(x^{\prime}\right) \mathrm{d} V\left(x^{\prime}\right), \quad \forall x \in V,
\end{aligned}
$$

where $\sigma_{i j}$ is the nonlocal stress tensor, $\varepsilon_{i j}$ is the strain tensor, $C_{i j k l}$ is the elastic modulus tensor in classical isotropic elasticity, $u_{i}$ is the displacement vector, $\alpha\left(\left|x-x^{\prime}\right|, \tau\right)$ is the nonlocal modulus or attenuation function incorporating the nonlocal effects into the constitutive equations. The quantity $\left|x-x^{\prime}\right|$ is the Euclidean distance. In $\tau=e_{0} a / l, e_{0}$ is a nonlocal scaling parameter, which has been assumed as a constant appropriate to each material, $a$ is an internal characteristic length, e.g., length of $\mathrm{C}-\mathrm{C}$ bond $(0.142 \mathrm{~nm})$ in carbon nanotube, granular distance, etc., and $l$ is an external characteristic length, e.g., wavelength, crack length, size of the sample, etc. and $V$ is the region occupied by the body.

The cylindrical coordinates system used for describing the wave propagation in the tubes is defined in Fig. 1. The $x$ coordinate is taken in the axial direction of the shell, where the $\phi$ and $z$ coordinates are in the circumferential and radial directions, respectively (Fig. 1). The displacements of the nanotube are defined by $u_{x}, u_{\phi}$

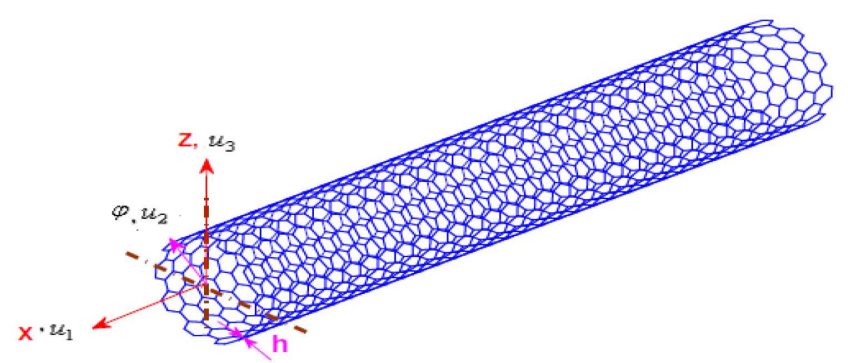

Fig. 1. Geometry of the tube with coordinate system.

and $u_{z}$ in the direction of $x, \phi$ and $z$ axes, respectively. The coordinates $u_{x}$ and $u_{\phi}$ represent in-plane axial and circumferential displacements of the tube wall midsurface, respectively, and $u_{z}$ represents the out-of-plane transverse displacement of the tube wall.

It is observed from Eq. (1) that not only the strain state of the reference location $x$ has the influence on the stress state at $x$, but also the strain state at $x^{\prime}$ can affect the stress state of the same location. It should be noted that because the spatial integrals are involved in Eq. (1), which result in the difficulty for solving the nonlocal problem. However, these integrals equations can be reduced to the partial differential forms under certain conditions with physically admissible kernels [25]. For the partial differential forms, the nonlocal constitutive relation can be expressed as [41]:

$$
\begin{aligned}
& \sigma_{x}-\left(e_{0}^{2} a^{2}\right) \nabla^{2} \sigma_{x}=\frac{E}{1-v^{2}}\left(\varepsilon_{x}+v \varepsilon_{\phi}+v \varepsilon_{z}\right), \\
& \sigma_{\phi}-\left(e_{0}^{2} a^{2}\right) \nabla^{2} \sigma_{\phi}=\frac{E}{1-v^{2}}\left(\varepsilon_{\phi}+v \varepsilon_{x}+v \varepsilon_{z}\right), \\
& \sigma_{z}-\left(e_{0}^{2} a^{2}\right) \nabla^{2} \sigma_{z}=\frac{E}{1-v^{2}}\left(\varepsilon_{z}+v \varepsilon_{\phi}+v \varepsilon_{x}\right), \\
& \sigma_{x \phi}-\left(e_{0}^{2} a^{2}\right) \nabla^{2} \sigma_{x \phi}=\frac{E}{2(1+v)} \varepsilon_{x \phi}, \\
& \sigma_{\phi z}-\left(e_{0}^{2} a^{2}\right) \nabla^{2} \sigma_{\phi z}=\frac{E}{2(1+v)} \varepsilon_{\phi z}, \\
& \sigma_{z x}-\left(e_{0}^{2} a^{2}\right) \nabla^{2} \sigma_{z x}=\frac{E}{2(1+v)} \varepsilon_{z x},
\end{aligned}
$$

where $\nabla^{2}=\frac{\partial}{\partial x^{2}}+\frac{\partial}{\partial y^{2}}$ is the Laplace operator for the Cartesian coordinates, $E$ and $\nu$ are the elastic modulus and Poisson's ratio of grapheme sheet, respectively and $\sigma_{k l}$ is the shear strain. The parameter $e_{0} a$ is the scale coefficient revealing the small-scale effect on the responses of structures of nanosize. If the small scalar parameter $a$ vanishes, Eqs. (2)-(8) will revert to Hooke's law of classical elasticity for a planar stress problem.

The constitutive equations of the nanotube in the polar coordinate system $(r, \phi)$, described by Erigena's nonlocal elasticity theory, are expressed as [41]:

$$
\sigma_{x}-\left(e_{0}^{2} a^{2}\right) \nabla^{2} \sigma_{x}=\frac{E}{1-v^{2}}\left(\varepsilon_{x}+v \varepsilon_{\phi}\right),
$$




$$
\begin{gathered}
\sigma_{\phi}-\left(e_{0}^{2} a^{2}\right) \nabla^{2} \sigma_{\phi}=\frac{E}{1-v^{2}}\left(\varepsilon_{\phi}+v \varepsilon_{x}\right), \\
\sigma_{\phi x}-\left(e_{0}^{2} a^{2}\right) \nabla^{2} \sigma_{\phi x}=\frac{E}{2(1+v)} \gamma_{\phi x}, \\
\sigma_{x \phi}-\left(e_{0}^{2} a^{2}\right) \nabla^{2} \sigma_{x \phi}=\frac{E}{2(1+v)} \gamma_{x \phi},
\end{gathered}
$$

where $\nabla^{2}=\frac{\partial}{\partial x^{2}}+\frac{\partial}{R^{2} \partial \phi^{2}}$ is the Laplace operator for the polar coordinate system and $\sigma_{x} \sigma_{\phi}$ and $\sigma_{x \phi}$ are the normal and shear stresses,

$$
\begin{aligned}
\varepsilon_{x} & =\frac{\partial u_{x}}{\partial x}, \quad \varepsilon_{\phi}=\frac{1}{R}\left(\frac{\partial u_{\phi}}{\partial x}+u_{z}\right), \\
\varepsilon_{i j} & =\frac{1}{R} \frac{\partial u_{i}}{\partial j}+\frac{\partial u_{j}}{\partial i}
\end{aligned}
$$

are the normal and shear strains. $R$ is the radius measured from the mid-plane of the cross-section in the following SWCNT analysis. Furthermore, there is another expression for the nonlocal continuum theory which is called the strain gradient form [41]. In this model, the gradient is exerted on the strain term. As the pioneer work, Peddieson et al. [42] developed the nonlocal form of the Euler-Bernoulli beam and predicted the feasibility of the nonlocal continuum theory for nanostructures. Sudak [43] used the nonlocal model to study the buckling behaviors of nanotubes. From then on, there is a growing interest of nonlocal continuum theory for the nanosystems.

\section{Transverse wave in SWCNTs}

Since 1D nanostructure are concerned, it is reasonable to set $\sigma_{\phi}=\sigma_{x \phi}=0$, and the constitutive Eqs. (9)-(12) reduce to

$$
\sigma_{x}-\eta^{2} \frac{\partial^{2} \sigma_{x}}{\partial x^{2}}=\frac{E}{1-v^{2}} \frac{\partial u_{x}}{\partial x}
$$

where $\eta=e_{0} a$.

For the transverse wave propagation in singlewalled carbon nanotubes, following the usual hypothesis of displacements [44]:

$$
u_{x}=-z \frac{\partial u_{z}}{\partial x} \text {. }
$$

Using (15) the constitutive Eqs. (9)-(12), and for the transverse wave propagation in single-walled carbon nanotubes, take the forms

$$
\sigma_{x}-\eta^{2} \frac{\partial^{2} \sigma_{x}}{\partial x^{2}}=\frac{-z E}{1-v^{2}} \frac{\partial^{3} u_{z}}{\partial x^{3}}
$$

The developed dynamic equation of motion of the wave propagation in the $x$-direction in SWCNTs with initial stress takes the form

$$
\frac{\partial Q}{\partial x}-\delta_{0} \frac{\partial^{2} u_{z}}{\partial x^{2}}=\rho h \frac{\partial^{2} u_{z}}{\partial t^{2}},
$$

where $Q$ is the shear resultant, $h$ is the thickness of the SWCNT, $\rho$ is the mass density and $\delta_{0}$ is an initial stress parameter, which takes positive value for compressive loading and negative for tensile loading.
The shear resultant, which is obtained from the integration of the stress over the thickness of shell, is given by Hooke's law

$$
Q=h \frac{\partial \sigma_{x}}{\partial x} .
$$

Substitution from Eq. (18) into Eq. (17) the wave motion equation can be derived as the following form:

$$
\frac{\partial^{2} \sigma_{z}}{\partial x^{2}}-\frac{\delta_{0}}{h} \frac{\partial^{2} u_{z}}{\partial x^{2}}=\rho \frac{\partial^{2} u_{z}}{\partial t^{2}} .
$$

Now eliminating $\sigma_{x}$ from (16) and (19) leads to

$$
\begin{gathered}
\frac{E}{1-v^{2}} \frac{\partial^{4} u_{z}}{\partial x^{4}}-\frac{\delta_{0}}{h}\left(\eta^{2} \frac{\partial^{4} u_{z}}{\partial x^{4}}-\frac{\partial^{2} u_{z}}{\partial x^{2}}\right) \\
-\rho\left(\eta^{2} \frac{\partial^{4} u_{z}}{\partial t^{2} \partial x^{2}}-\frac{\partial^{2} u_{z}}{\partial t^{2}}\right)=0 .
\end{gathered}
$$

This is the final governing equation for transverse waves propagating in SWCNTs under initial stress.

\section{Frequency equation}

In our study here, the $\phi$-directional displacement $u_{\phi}$ is assumed to be zero and the harmonic solution of the displacement could be written as [44]:

$$
u_{z}(x, t)=U \exp (\mathrm{i}(k x-\omega t)),
$$

where $\mathrm{i}=\sqrt{-1}, U$ is the amplitude of the displacement, $k$ is the wave number of the transverse wave and $\omega$ is the circular frequency of the transverse waves. The governing Eq. (20) becomes then

$$
\begin{aligned}
& k^{2}\left[\left(\frac{k^{2} E}{1-v^{2}}\right)-\frac{\delta_{0}}{h}\left(\eta^{2} k^{2}+1\right)\right] \\
& -\rho \omega^{2}\left(\eta^{2} k^{2}+1\right)=0 .
\end{aligned}
$$

Solving the resulting frequency equation, the phase velocity, $C_{S}=\frac{\omega}{k}$, can be found to be

$$
C_{S}=\sqrt{\frac{k^{2} E}{\rho\left(1-v^{2}\right) \Omega}-\frac{\delta_{0}}{\rho h}},
$$

where $\Omega=\left(1+\eta^{2} k^{2}\right)$.

Obviously, transverse waves are present for initial tensile loading $\delta_{0}<0$. On the contrary, for an initial compressive loading, i.e., $\delta_{0}>0$, waves may not exist unless the compressive loading $\delta_{0}$ is sufficiently small, satisfying

$$
\frac{k^{2} E}{\rho\left(1-v^{2}\right) \Omega}-\frac{\delta_{0}}{\rho h}>0 \Rightarrow \delta_{0}<\frac{k^{2} E h}{\left(1-v^{2}\right) \Omega} .
$$

Moreover, from Eq. (23) it is clear that the relation between $\omega$ and $k$ is nonlinear, which results from scale coefficient $\eta$, then the transverse waves in SWCNTs become dispersive, although this kind of elastic wave is non-dispersive in the classic continuum theory [45].

Alternatively, solving Eq. (24), we obtain

$$
k>\sqrt{\frac{\delta_{0}\left(1-v^{2}\right) \Omega}{E h}} .
$$

Then, we conclude from above equation, for elastic SWCNTs subjected to initial compressive stress, the transverse waves could be propagated only for waves 
with larger wave numbers or cannot propagate for those below a critical value of the wave number.

Consider a special case when the small scalar parameter $a$ vanishes $(\eta=0 \Rightarrow \Omega=1)$. This represents the case of classical elasticity. Then the above result reduces to

$$
k>\sqrt{\frac{\delta_{0}\left(1-v^{2}\right)}{E h}} .
$$

Furthermore, the group velocity, $C_{g s}=\frac{d \omega}{d k}$, can be obtained as

$$
\begin{aligned}
C_{g s} & =\sqrt{\frac{E k^{2}}{\rho\left(1-\nu^{2}\right) \Omega}-\frac{\delta_{0}}{\rho h}} \\
\times & {\left[1+\frac{E h k^{2}}{E h k^{2} \Omega-\delta_{0}\left(1-\nu^{2}\right) \Omega^{2}}\right] . }
\end{aligned}
$$

\section{Results and discussion}

In order to realize the role of important parameters on the transverse waves propagation in a single-walled carbon nanotubes, some instructive parametric studies are carried out. In this regard, the effects of small scale and initial stress on the dynamic response of SWCNTs are studied in some detail. For this purpose, the Young modulus $E=1 \mathrm{TPa}$, the mass density $\rho=1340 \mathrm{~kg} / \mathrm{m}^{3}$ and the Poisson ratio $\nu=0.27$ [35] and the SWCNT was the $(40,0)$ zigzag tube with an effective diameter of $3.13 \mathrm{~nm}$.

The most remarkable feature in Fig. 2 is that when the wave number is smaller than $1 \mathrm{~nm}^{-1}$, the dispersion relations for different scale coefficients seem to be zero. Compared with the corresponding case without the nonlocal effect (i.e., $\eta=0$ ), which is viewed as the small scale effect, will arise gradually with the increases of wave number $k$. The higher the mode is, the higher the scale effect can be observed. Also, comparisons show that the change tendency of the frequency of transverse waves in SWCNTs with initial compression stresses (b) and tensile initial stress (c) is very small. It can be seen from Fig. 2 that the frequency value estimated by the local elastic theory is higher than that by the nonlocal continuum model. For almost all of the wave numbers the frequency corresponding to $\eta=2 \mathrm{~nm}$ is the smallest. It implies that dispersion relation can be strengthened by increasing the scale coefficients. As a result, the small scale effects should be considered in the wave propagation properties in the carbon nanotubes. Moreover, the dispersion phenomenon of the transverse wave in pre-stressed carbon nanotubes (CNTs) is an interesting observation, which cannot be found in the classical model.

In order to illustrate the scale and initial stress effects on the transverse wave dispersion properties, the phase velocity $C_{s}$ and the group velocity $C_{g s}$ are computed and displayed in Figs. 3 and 4, respectively.

Figure 3 shows the relation between the phase velocity $C_{s}$ and the wave number $k\left(\mathrm{~m}^{-1}\right)$ for different values of scale coefficients $(\eta=0,1,2)$ and initial stress parameter
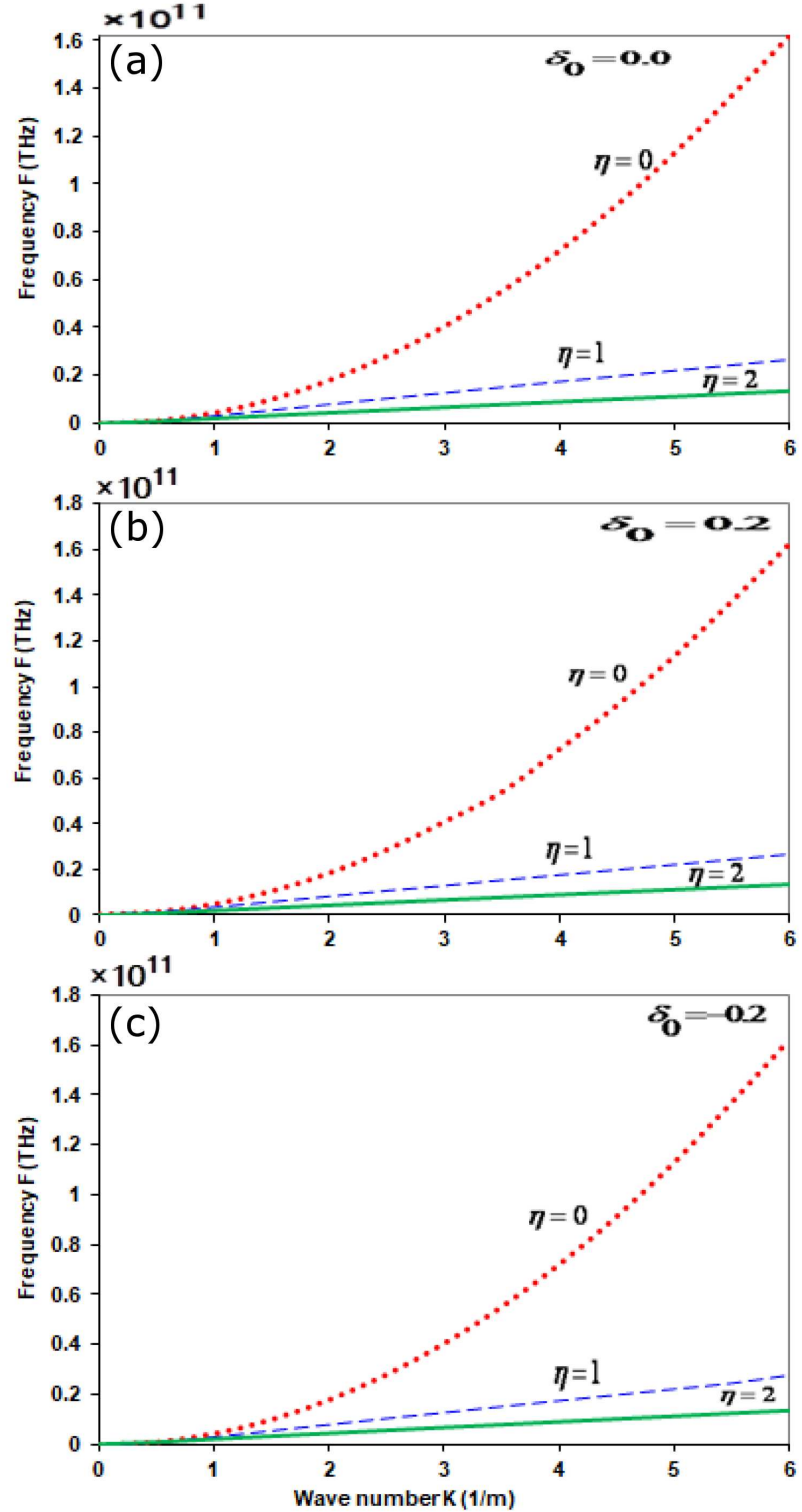

Fig. 2. Dispersion relation for single-wall carbon nanotubes for different values of scale coefficients $(\eta=0,1,2)$ and the initial stress parameter (a) $\delta_{0}=0.0$, (b) $\delta_{0}=0.2$, and (c) $\delta_{0}=-0.2$.

(a) $\delta_{0}=0.0$, (b) $\delta_{0}=0.2$, and (c) $\delta_{0}=-0.2$. The most remarkable feature in Fig. 3 is that the value of the phase velocity is the same for $\eta=0$ at $\delta_{0}=0.0,0.2,-0.2$. However, the phase velocity decreases with the wave number increasing. It means that the classical continuum theory is not accurate to predict the mechanical response of CNTs. It is also clear from Fig. 3 that when the initial stress in SWCNTs is the compressive stress (b), transverse phase velocity increases gradually starting from lower frequency $\left(k=0 \mathrm{~nm}^{-1}\right)$, and when the initial stress in SWCNTs is the tensile stress (c), transverse phase velocity increases gradually starting from higher frequency $\left(k=0.5 \mathrm{~nm}^{-1}\right)$. 

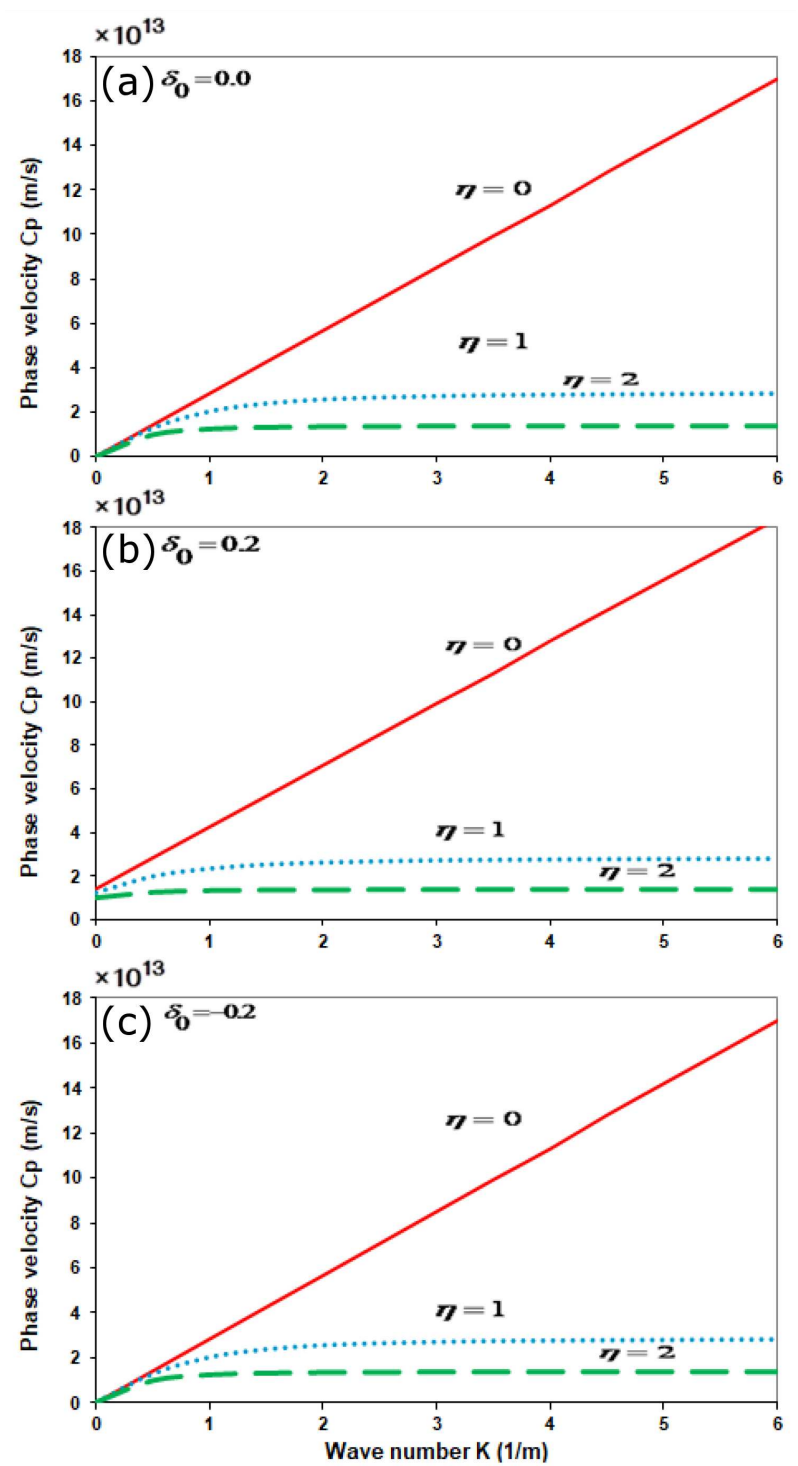

Fig. 3. Phase velocity $C_{s}$ vs. wave number for different values of scale coefficients $(\eta=0,1,2)$ and the initial stress parameter (a) $\delta_{0}=0.0$, (b) $\delta_{0}=0.2$, and (c) $\delta_{0}=-0.2$.

Figure 4 shows the relation between the group velocity $C_{g s}$ and the wave number $k(1 / \mathrm{m})$ for different values of scale coefficients $(\eta=0,1,2)$ and the initial stress parameter (a) $\delta_{0}=0.0$, (b) $\delta_{0}=0.2$, and (c) $\delta_{0}=-0.2$. Comparisons show that the change is clearly observed in the case of $\delta_{0}=0.2$. From Fig. 4 one can see that when the initial stress in SWCNTs is the tensile stress (c), transverse group velocity increases gradually starting from lower frequency $\left(k=0 \mathrm{~nm}^{-1}\right)$, and when the initial stress in SWCNTs is the compressive stress (b), transverse phase velocity increase gradually starting from higher frequency $\left(k=0.5 \mathrm{~nm}^{-1}\right)$.

From Figs. 3 and 4 it is necessary to take the nonlocal model into account to investigate the wave characteristics in CNTs, especially for larger wave numbers.
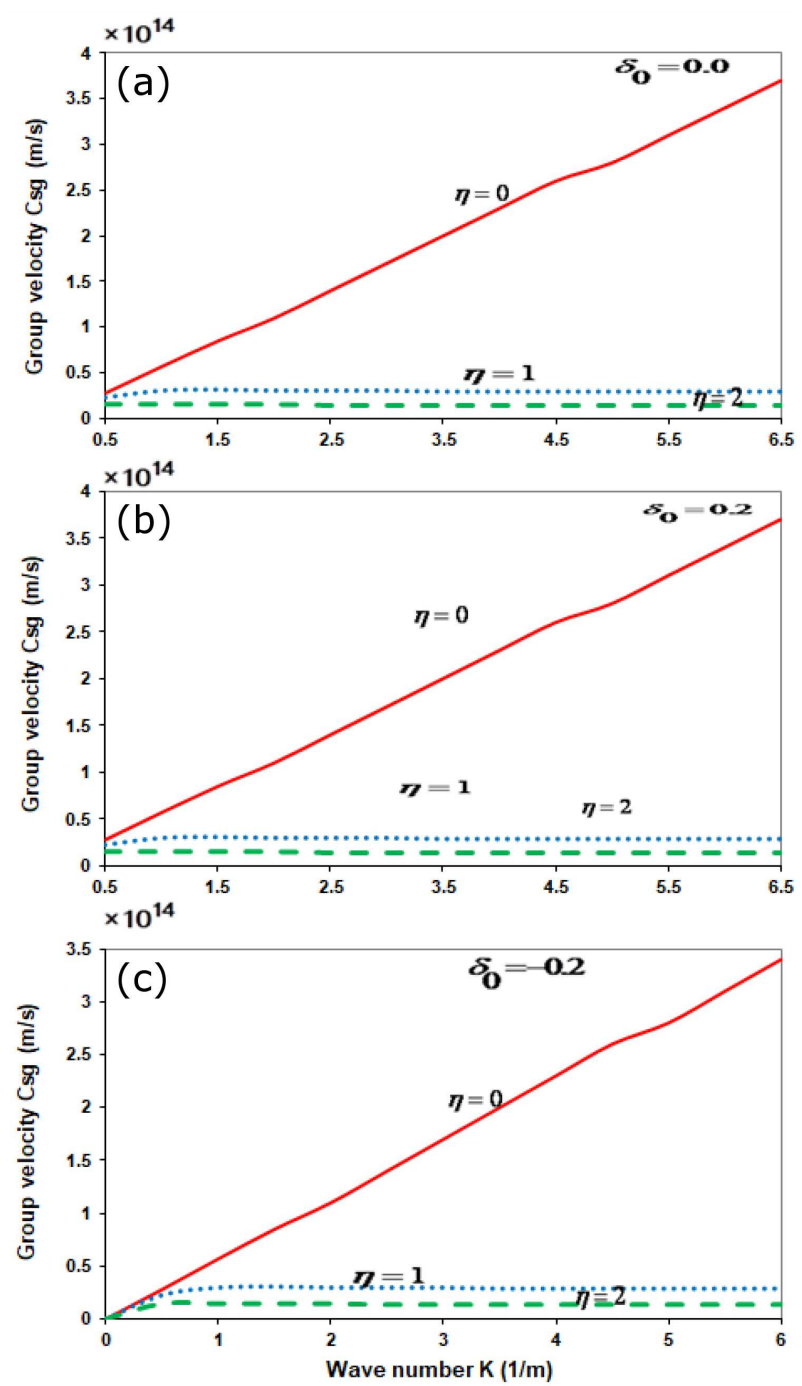

Fig. 4. Group velocity $C_{g s}$ vs. wave number for different values of scale coefficients $(\eta=0,1,2)$ and the initial stress parameter (a) $\delta_{0}=0.0$, (b) $\delta_{0}=0.2$, and (c) $\delta_{0}=-0.2$

The phase velocity and the group velocity versus the scale coefficient with the nonlocal model are presented in Figs. 5-7. The wave number $k$ used in the calculation is $0.1 \times 10^{9}, 1 \times 10^{9}$ and $5 \times 10^{9} 1 / \mathrm{m}$. From Fig. 5 it can be seen that the values of phase velocity and group velocity are almost the same for smaller wave numbers (e.g. $\left.k=0.1 \times 10^{9} 1 / \mathrm{m}\right)$ at $\delta_{0}=0.0$, which implies that the scale and initial stress effects are not obvious. However, these effects become remarkable with the wave number becoming larger in Figs. 6 and 7. Both of the velocities decrease as the scale coefficient increases. In Figs. 5-7 the phase and group velocities decrease to zero when the scale coefficient is about $1 \mathrm{~nm}$ at tensile initial stress (c). Comparisons show that the effect of initial stress is clearly observed in the case of $\delta_{0}=0.2$ (compression initial stress) for $k=0.1 \times 10^{9}, 1 \times 10^{9}$ (lower frequencies). 

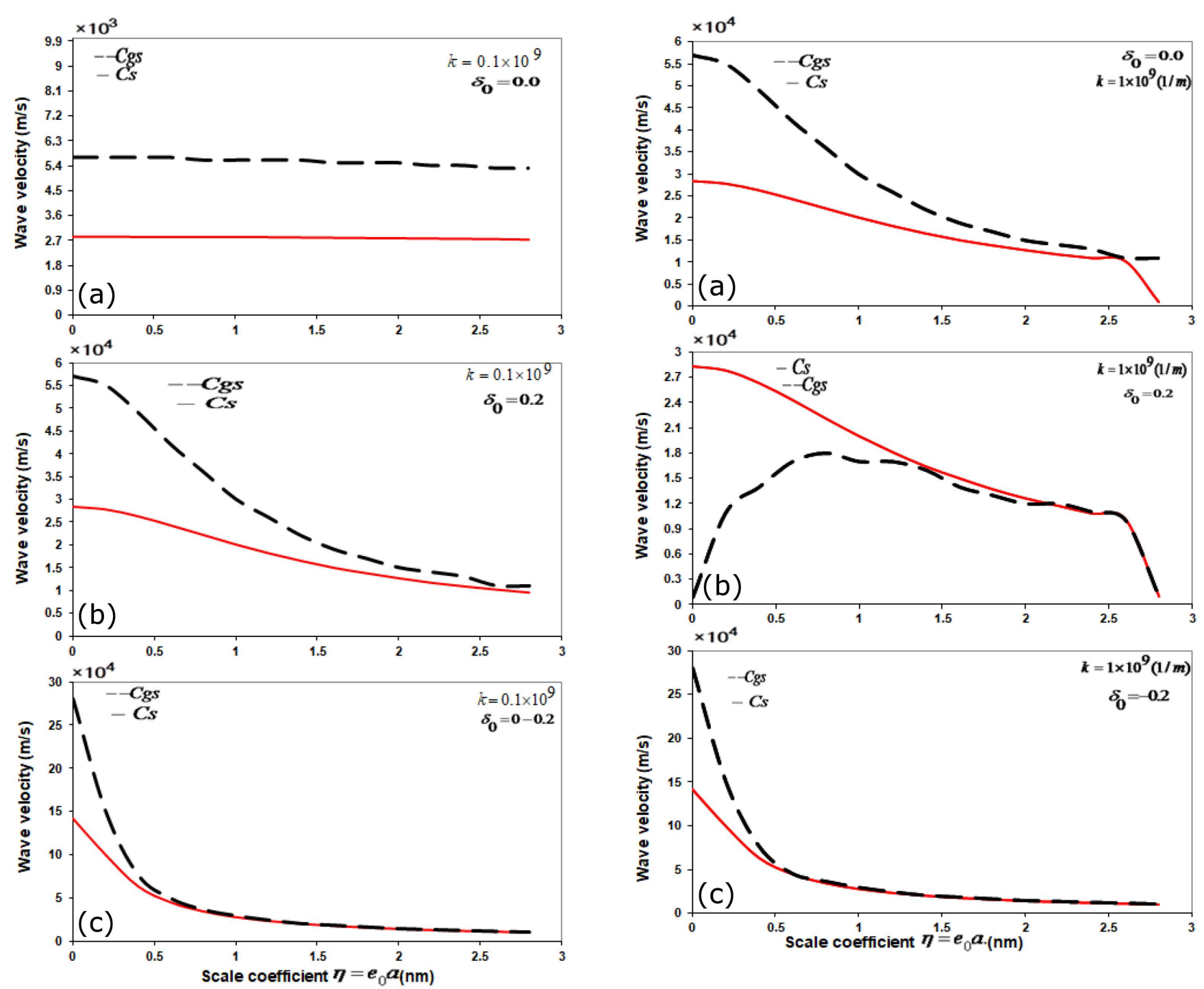

Fig. 5. Phase velocity $C_{s}$ and group $C_{g s}$ velocities vs. scale coefficient with wave numbers $k=0.1 \times 10^{9} 1 / \mathrm{m}$ for different values of the initial stress parameter (a) $\delta_{0}=0.0$, (b) $\delta_{0}=0.2$, and (c) $\delta_{0}=-0.2$.

\section{Conclusion}

The main contribution in this work is to describe the scale and initial stress effects on the transverse wave speeds in SWCNTs based on the nonlocal elastic theory. Explicit formulas are derived for predicting the scale and initial stress effects of SWCNTs based on nonlocal elastic theory. The expressions of the phase velocity and the group velocity are presented. The numerical simulations are performed with the consideration of small scale and initial stress effects. We conclude that the classical continuum theory is not suitable and the nonlocal continuum model should be employed to investigate the transverse wave characteristics in SWCNTs. The transverse wave propagation in pre-stressed single-wall carbon nanotubes becomes dispersive, which results from the small

Fig. 6. Phase velocity $C_{s}$ and group $C_{g s}$ velocities vs. scale coefficient with wave numbers $k=1 \times 10^{9} 1 / \mathrm{m}$ for different values of the initial stress parameter (a) $\delta_{0}=0.0$, (b) $\delta_{0}=0.2$, and (c) $\delta_{0}=-0.2$.

scale effects. The dispersion degree can be strengthened by increasing the scale coefficient. The scale effects on the phase and group velocities become more remarkable for larger wave numbers. Characteristics of the transverse waves propagation in SWCNTs were discussed, and obtained results turn out that the phase and group velocities are sensitive to initial stress for higher frequencies. As a result, for elastic SWCNTs subjected to initial compressive stress, transverse waves can propagate only for waves with larger wave numbers or cannot propagate for those below a critical value of the wave number. The effects of initial stress on the transverse wave propagation in the SWCNTs may result in some complex dynamic phenomena of terahertz sound waves propagation in the SWCNTS, which can be used as a useful reference for the designs of nanoelectronic and nanodrive devices, nanooscillators, and nanosensors. 

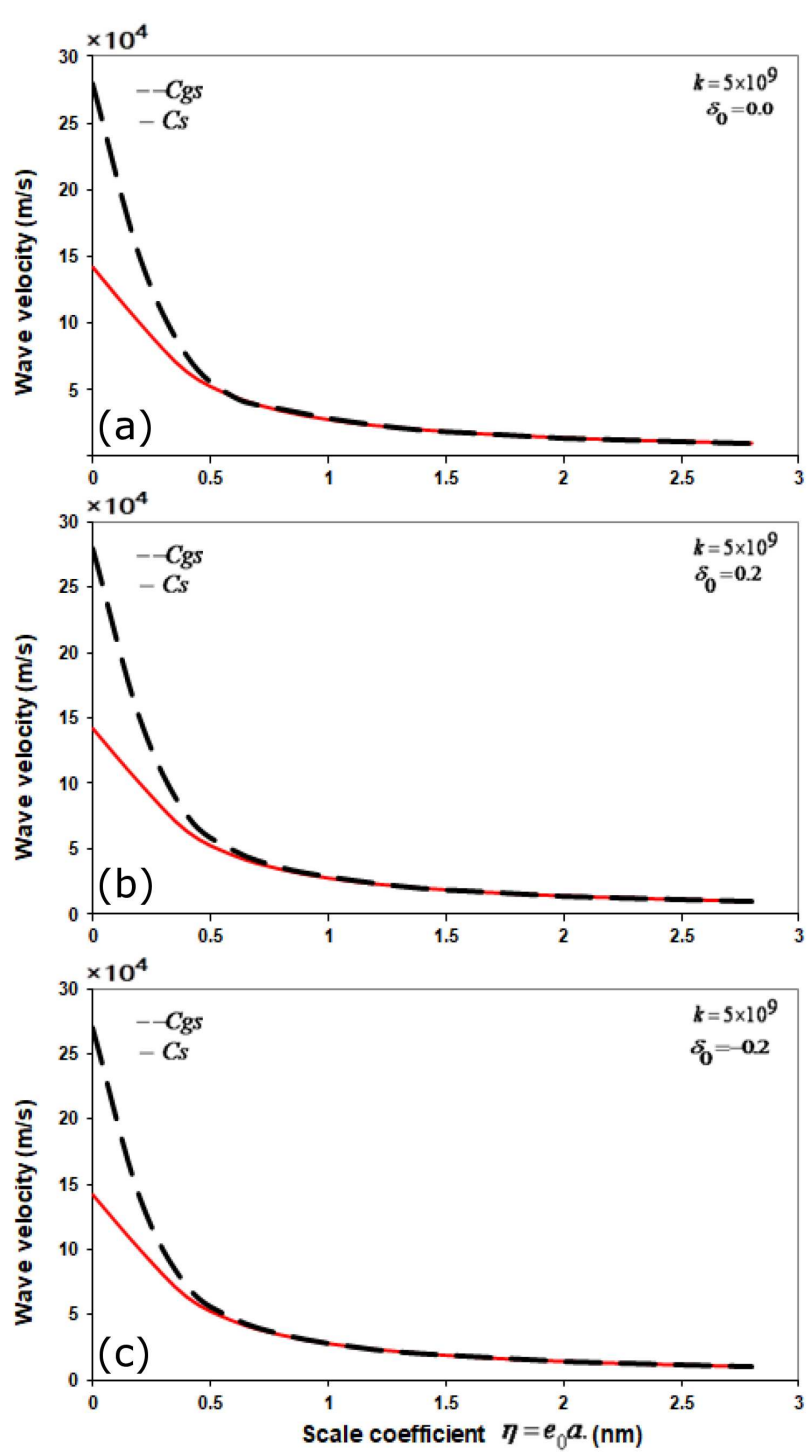

Fig. 7. Phase velocity $C_{s}$ and group $C_{g s}$ velocities vs. scale coefficient with wave numbers $k=5 \times 10^{9} 1 / \mathrm{m}$ for different values of the initial stress parameter (a) $\delta_{0}=0.0$, (b) $\delta_{0}=0.2$, and (c) $\delta_{0}=-0.2$.

\section{Acknowledgments}

The author wishes to acknowledge support provided by the Deanship of Scientific Research of Prince Sattam Bin Abdulaziz University.

\section{References}

[1] E.T. Thostenson, Z. Ren, T.W. Chou, Comp Sci. Tech. 61, 1899 (2001).

[2] L.S. Schadler, S.C. Giannaris, P.M. Ajayan, Appl. Phys. Lett. 73, 3842 (1998).

[3] L. Vaccarini, C. Goze, L. Henrard, E. Hernandez, P. Bernier, A. Rubio, Carbon 381681 (1999).

[4] M.F. Yu, O. Lourie, M.J. Dyer, K. Moloni, T.F. Kelly, R.S. Ruoff, Science 287, 637 (2000).
[5] S.B. Nadarajan, P.D. Katsikis, E.S. Papazoglou, Appl. Phys. A 2, 437 (2007).

[6] A.B. Dalton, S. Collins, J. Razal, E. Munoz, V.H. Ebron, B.G. Kim, J. Mater. Chem. 14, 1 (2004).

[7] S. Iijima, Nature 354, 6 (1991).

[8] P. Ball, Nature 414, 142 (2001).

[9] R.H. Baughman, A.A. Zakhidov, W.A. de Heer, Science 297, 787 (2002).

[10] S. Iijima, C. Brabec, A. Maiti, J. Bernholc, Chem. Phys. 104, 2089 (1996).

[11] E. Hernandez, C. Goze, P. Bernier, A. Rubio, Phys. Rev. Lett. 80, 4502 (1998).

[12] C.Y. Li, T.W. Chou, Phys. Rev. B 73, 245407 (2006).

[13] B.I. Yakobson, M.P. Campbell, C.J. Brabec, J. Bernholc, Comput. Mater. Sci. 8, 341 (1997).

[14] K.M. Liew, X.Q. He, C.H. Wong, Acta Mater. 52 $2521(2004)$

[15] B.I. Yakobson, M.P.Campbell, C.J. Brabec, J. Bernholc, Comput. Mater. Sci. 8, 341 (1997).

[16] A. Krishnan, E. Dujardin, T.W. Ebbesen, P.N. Yianilos, M.M. Treacy, Phys. Rev. B 58, 14013 (1998).

[17] C.Q. Ru, Phys. Rev. B 62, 9973 (2000).

[18] C.Q. Ru, Phys. Rev. B 62, 10405 (2000).

[19] R. Parnes, A. Chiskis, J. Mech. Phys. Solids 50, 855 (2002).

[20] E.W. Wong, P.E. Sheehan, C.M. Lieber, Science 277, 1971 (1997)

[21] Q. Lourie, D.M. Cox, H.D. Wagner, Phys. Rev. Lett. 81, 1638 (1998).

[22] C.Y. Wang, C.Q. Ru, A. Mioduchowski, Phys. Rev. $B$ 72, 075414 (2005).

[23] T. Natsuki, M. Endo, H. Tsuda, J. Appl. Phys. 99 , 034311 (2006).

[24] A.C. Eringen, D.G.B. Edelen, Int. J. Eng. Sci. 10 233 (1972).

[25] A.C. Eringen, J. Appl. Phys. 54, 4703 (1983).

[26] A.C. Eringen, Non-local Polar Field Models, Academic, New York 1996.

[27] Y.-Z. Wang, F.-M. Li, K. Kishimoto, Physica E 42 1356 (2010).

[28] X. Wang, H. Cai, Acta Mater. 54, 2067 (2006).

[29] H. Cai, X. Wang, Nanotechnol. 17, 45 (2006).

[30] Y. Zhang, G. Liu, X. Han, Phys. Lett. A 340, 258 (2005).

[31] P. Lu, H.P. Lee, C. Lu, H.B. Chen, Int. J. Mech. Sci. 50, 501 (2008).

[32] C.M. Wang, W.H. Duan, J. Appl. Phys. 104, 014303 (2008).

[33] H. Heireche, A. Tounsi, A. Benzair, Nanotechnol. 19, 185703 (2008).

[34] M. Mitra, S. Gopalakrishnan, Computat. Mater. Sci. 45, 411 (2009).

[35] M.M. Selim, Acta Phys. Pol. A 135, 903 (2019).

[36] M.M. Selim, Brazil. J. Phys. 40, 283 (2010).

[37] M.M. Selim, Acta Phys. Pol. A 119, 778 (2011). 
[38] Z.G. Zhou, B. Wang, Int. J. Solids Struct. 43, 887 (2006).

[39] R. Artan, M. Omurtag, Int. J. Eng. Sci. 38, 395 (2000).

[40] S. Ghosh, A. Kumar, V. Sundararaghavan, A.M. Waas, Int. J. Solids Struct. 50, 2837 (2013).

[41] Q. Wang, C.M. Wang, Nanotechnol. 18, 075702 (2007).
[42] J. Peddieson, G.R. Buchanan, R.P. McNitt, Int. J. Eng. Sci. 41, 305 (2003).

[43] L.J. Sudak, J. Appl. Phys. 94, 7281 (2003).

[44] J.-X. Wu, X.-F. Li, G.-J. Tang, Physica B 407, 684 (2012).

[45] J.D. Achenbach, Wave Propagation in Elastic Solids, North-Holland, Amsterdam 1973. 